\title{
INCIDENCIA DE HEMORRAGIA POSPARTO CON BASE EN EL USO TERAPÉUTICO DE UTEROTÓNICOS. RESULTADOS MATERNOS EN UN HOSPITAL DE MEDIANA COMPLEJIDAD DE BOGOTÁ, COLOMBIA, $2016^{1}$
}

\section{Incidence of postpartum hemorrhage based on} the use of uterotonics. Maternal outcomes in an Intermediate Complexity Hospital in Bogotá, Colombia, 2016

\author{
Luis Felipe López-García MD², Diana Paola Ruiz-Fernández $M D^{3}$, \\ Carlos Germán Zambrano-Cerón MD ${ }^{3}$, Jorge Andrés Rubio-Romero MD, $\mathrm{MSc}^{4}$ \\ Recibido: febrero 10/17 - Aceptado: septiembre 25/17
}

\section{RESUMEN}

Objetivo: describir la incidencia de hemorragia obstétrica posparto (HPP) severa, definida por el uso de medicamentos uterotónicos, como también las intervenciones realizadas y los desenlaces maternos en las primeras 24 horas.

Materiales y métodos: serie de casos de gestantes que presentaron HPP luego de parto vaginal o cesárea entre el 1 de febrero y el 31 de octubre de 2016, en una institución pública de mediana complejidad en Bogotá. Muestreo consecutivo. Se midieron las características sociodemográficas, clínicas y los factores de riesgo para HPP. Se determinó la causa de la hemorragia, grado de choque, sangrado posparto estimado, activación del código

1 Trabajo presentado para optar al título de Ginecoobstetra, Universidad Nacional de Colombia, Bogotá (Colombia).

2 Especialista en Obstetricia y Ginecología, Universidad Nacional de Colombia, Bogotá (Colombia)._luflopezga@unal.edu.co

3 Especialista en Obstetricia y Ginecología, Universidad Nacional de Colombia, Bogotá (Colombia).

4 Profesor titular, Departamento de Obstetricia y Ginecología, Universidad Nacional de Colombia, Bogotá (Colombia). rojo obstétrico, intervenciones realizadas, ingreso a unidad de cuidado intensivo, necesidad de transfusión sanguínea y mortalidad. Se realizó análisis descriptivo.

Resultados: de 1.633 nacimientos (1.080 partos y 553 cesáreas) se presentaron 35 (2,1\%) casos de HPP por uso de oxitócicos (26 y 9 respectivamente), y se activó el código rojo obstétrico en 11 casos (0,67\%). No hubo mortalidad materna. La principal causa de HPP fue hipotonía uterina 29/35 (82\%), y en el $82,8 \%$ de los casos se resolvió con manejo médico; 4/35 (11,4\%) requirió taponamiento uterino adicional para control del sangrado; 2/35 (5,6\%) de las mujeres requirió manejo quirúrgico: sutura hemostática 1/35 (2,8\%) e histerectomía $1 / 35(2,8 \%)$.

Conclusiones: la identificación de HPP severa por criterios más reproducibles para iniciar el tratamiento oportuno podría ser más efectiva y segura en cuanto a resultados maternos.

Palabras clave: hemorragia posparto, parto, taponamiento uterino con balón, evaluación de resultados. 


\section{ABSTRACT}

Objective: To describe the incidence of obstetric postpartum haemorrhage (PPH), defined by the use of uterotonic drugs, as well as the interventions performed, and maternal outcomes in the first 24 hours.

Materials and methods: A series of cases of pregnant women who presented PPH after vaginal delivery or cesarean section between February 1 and October 31, 2016, in a public intermediate complexity institution in Bogotá. Consecutive sampling was used. Sociodemographic, clinical and risk factors for PPH were measured. Variables measured were the cause of PPH, the degree of shock, estimated postpartum bleeding, frequency of activation of the "obstetric red code," interventions performed, admission to an intensive care unit, blood transfusions, and maternal mortality. A descriptive analysis was performed.

Results: Out of 1633 births (1080 deliveries and 553 cesareans), 35 cases (2.1\%) (26 and 9 cases respectively) were identified as HPP by the uses of and the uterotonic drugswere identified and the "obstetric red code" was activated in 11 cases $(0.67 \%)$. There was no maternal mortality. The main cause of PPH was uterine hypotonia in 29/35 (82\%) and $82.8 \%$ of cases resolved with medical treatment. 4/35 (11.4\%) required uterine tamponade to control bleeding. 2/35 (5.6\%) of the women required surgical management: 1/35 (2.8\%) hemostatic sutures and 1/35 (2.8\%) hysterectomy.

Conclusions: The identification of severe PPH by more reproducible criteria for initiating timely treatment could be more effective and safer in terms of maternal outcomes.

Key words: Pospartum hemorrhage, delivery, uterine ballon tamponade, treatment outcome.

\section{INTRODUCCIÓN}

La hemorragia posparto primaria (HPP) es definida tradicionalmente como la pérdida sanguínea estimada mayor de $500 \mathrm{cc}$ en las primeras 24 horas cuando es un parto vaginal, y mayor de 1000 cc después de una cesárea (1). Se presenta en el 2-4\% de los partos y el 6-7\% de las cesáreas. En el 1\% de los casos se trata de hemorragia severa que corresponde a una pérdida sanguínea mayor de 1.000 cc (2). Es la causa más frecuente de mortalidad (19\%) y morbilidad materna en el mundo: en los países desarrollados produce el $8 \%$ de las muertes, mientras que en los países en vía de desarrollo es responsable del 19,7 \% de las muertes maternas (3). En Colombia es responsable del $23 \%$ de las muertes maternas (4). La prevención y el manejo de la hemorragia primaria posparto son fundamentales para disminuir la mortalidad materna (5). Las causas de la hemorragia obstétrica luego del nacimiento se han agrupado en cuatro grupos principales y se han denominado: tono, trauma, tejido y trombina, siendo la más frecuente la atonía uterina en un 70\% (6-9).

La hemorragia obstétrica severa luego del parto constituye una emergencia vital, clasificada como morbilidad materna extrema, y su notificación es obligatoria para el sistema de vigilancia epidemiológica en Colombia (10). Dado que la estimación visual de la pérdida sanguínea es poco fiable para hacer el diagnóstico de HPP severa, y tiende a subestimar la magnitud del sangrado, se han propuesto varios métodos basados en los hallazgos clínicos más precisos para estimar la cantidad de pérdida sanguínea (11). Todos estos buscan desencadenar una serie de acciones secuenciales, coordinadas y oportunas realizadas por un equipo interdisciplinario cuyo objetivo central es disminuir la morbi-mortalidad materna originada por esta causa. Estas acciones son conocidas en Colombia como "código rojo obstétrico" (11). Como acción inicial a esta aproximación de manejo se busca hacer un primer diagnóstico de la presencia de choque cardiovascular. Este algoritmo de manejo incorpora las recomendaciones incluidas en la guía de la Organización Mundial de la Salud (OMS) (5) y del Royal College of Gynaecology and Obstetrics (12). La instauración efectiva de estas acciones demanda la activación de un equipo de respuesta rápida y la destinación prioritaria de los recursos hospitalarios hasta que se logre la resolución del cuadro. 
El retraso en la identificación de la HPP severa es la principal causa de morbi-mortalidad materna secundaria a hemorragia $(11,13,14)$. El enfoque diagnóstico y terapéutico de "código rojo obstétrico", aunque efectivo para el control del sangrado y la mejora en la sobrevida de las pacientes una vez es activado, resulta poco sensible para la identificación y notificación de todos los casos de hemorragia obstétrica primaria, ya que en algunos la presencia de HPP podría ser considerada como "no suficientemente" severa para ser notificada, lo que desencadena el manejo por parte del personal tratante, pero no conlleva a la activación formal a nivel institucional el código rojo obstétrico. Esta HPP "no suficientemente severa" podría estar asociada a morbilidad materna importante y riesgo de muerte, y a un subregistro y subnotificación de los casos de hemorragia a los sistemas de vigilancia epidemiológica en Colombia.

El presente trabajo tiene como objetivo hacer una exploración de la incidencia de hemorragia obstétrica primaria a partir de una definición más reproducible basada en el empleo de uno o más medicamentos uterotónicos, en dosis adicionales a las utilizadas rutinariamente para el manejo activo del alumbramiento, independiente de la activación del código rojo obstétrico, como también, describir las intervenciones realizadas para el control de dichas hemorragias y los desenlaces maternos del tratamiento instaurado en las primeras 24 horas hasta los 7 días posparto, y evaluar la magnitud de este subregistro.

\section{MATERIALES Y MÉTODOS}

Serie de casos de mujeres que presentaron hemorragia obstétrica primaria dentro de una cohorte de gestantes atendidas por parto vaginal o cesárea entre el 1 febrero y el 31 de octubre de 2016, en un hospital general de mediana complejidad, público, ubicado en Bogotá, que atiende pacientes del Sistema de Seguridad Social de Salud de Colombia (SSSC) y sirve de centro para la formación de estudiantes de pre y posgrado de medicina. Se excluyeron las mujeres con diagnóstico anteparto de ruptura uterina, acretismo placentario, placenta previa, trastornos de la coagulación o coagulación intravascular diseminada por tener indicación de tratamientos diferentes a la administración de medicamentos uterotónicos en presencia de HPP.

Se definieron como casos de hemorragia obstétrica a las mujeres que requirieron el empleo de medicamentos (oxitocina, metilergonovina o misoprostol) adicionales a la dosis de 10 unidades intravenosas de oxitocina usada para el manejo activo del alumbramiento durante parto o cesárea a criterio del médico responsable del servicio de obstetricia, y de acuerdo con los protocolos y las guías institucionales.

Se realizó muestreo consecutivo. Teniendo en cuenta la incidencia de hemorragia posparto reportada en la literatura, 2-4\% de los partos y 6-7\% de las cesáreas en estudios previos $(13,15)$ y la frecuencia reportada de 2.200 nacimientos por año en la institución, se estimó un número de 90 casos de hemorragia obstétrica distribuidos en 50 casos posparto vaginal y 40 casos poscesárea durante el periodo de observación, según la proporción histórica de cesáreas de la institución (16).

Procedimiento. Las pacientes fueron identificadas mediante búsqueda activa en la farmacia de la institución, por despacho de dosis de medicamentos uterotónicos disponibles (oxitocina, metilergonovina o misoprostol), adicionales a la dosis de 10 unidades de oxitocina empleadas para el manejo activo del alumbramiento, y en los registros de notificación de los casos de activación del código rojo obstétrico. Se revisaron las historias clínicas hasta el egreso y el control posparto al séptimo día, recogiendo la información en formatos específicos. Los datos se archivaron bajo llave en un área segura, con acceso limitado al personal del estudio, siendo digitados en una base de datos creada en Excel y analizada mediante EPIINFO 7.0, previa verificación de la calidad de los mismos.

Las variables por medir fueron: edad materna, presencia de antecedentes médicos, enfermedades 
asociadas al embarazo, paridad, antecedente de hemorragia posparto, edad gestacional al momento del parto en semanas, presencia de controles prenatales, concentración de hemoglobina $(\mathrm{g} / \mathrm{dL})$ del último control prenatal, altura uterina, inducción del trabajo de parto, vía del parto, episiotomía, desgarros perineales, peso y talla neonatal. Las variables de desenlace fueron la presencia de hemorragia obstétrica definida por el empleo de medicamentos (oxitocina, metilergonovina o misoprostol) adicionales a la dosis de 10 unidades de oxitocina empleada para el manejo activo del alumbramiento durante parto o cesárea en las primeras 24 horas posteriores al nacimiento, causa de la hemorragia (hipotonía, trauma, presencia de tejido o alteración de la coagulación posparto), grado de choque estimado según los criterios definidos en el protocolo de "código rojo" (11), sangrado posparto estimado y activación del equipo de código rojo obstétrico. Las intervenciones definidas para el control de la hemorragia obstétrica fueron: manejo médico (empleo de los uterotónicos descritos), manejo médico avanzado (uterotónicos e introducción de taponamiento uterino) y manejo quirúrgico (laparotomía para suturas uterinas hemostáticas o histerectomía). Los desenlaces maternos evaluados fueron mortalidad, histerectomía, ingreso a unidad de cuidado intensivo y necesidad de transfusión sanguínea.

Análisis estadístico. Las variables cuantitativas se resumen con medidas de tendencia central, medias o medianas, y medidas de dispersión según los resultados de las pruebas de normalidad y las variables cualitativas en números absolutos y proporciones.

Aspectos éticos. Este proyecto de investigación fue sometido a evaluación por el comité de ética de la Facultad de Medicina de la Universidad Nacional de Colombia, y aprobado en acta 143 del 10 de septiembre de 2015, y por el comité de ética del Hospital de Engativa ESE, el día 18 de diciembre de 2015.

\section{RESULTADOS}

Durante el periodo de estudio hubo 1.633 nacimientos: 1.080 partos vaginales y 553 cesáreas. para una proporción de cesáreas de 33,8 \%. Bajo el criterio de hemorragia obstétrica definido por el uso adicional de uterotónicos se identificaron un total de 35 casos, para una incidencia global de 2,1\% en el periodo. Según la vía del parto, la incidencia de hemorragia obstétrica posparto fue 2,4\% (26 casos) y 1,6\% (nueve casos) poscesárea. Basados en la notificación por la activación del código rojo obstétrico, la incidencia de hemorragia obstétrica posparto fue de $0,83 \%(9 / 1080)$ y poscesárea de $0,36 \%(2 / 553)$. No se presentaron muertes maternas. Las características demográficas de los casos identificados y las causas descritas de hemorragia obstétrica se muestran en la tabla 1. En 24/35 pacientes $(68,6 \%)$ se administraron medicamentos uterotónicos adicionales al manejo activo del alumbramiento, y no se realizó la activación del protocolo institucional de respuesta o código rojo obstétrico.

Seis pacientes tenían antecedentes médicos, de las cuales tres eran obesas, una presentó infección urinaria a repetición, una farmacodependencia y una hipertrigliceridemia. Ninguna paciente presentó anemia antes del parto. Las enfermedades asociadas a la gestación encontradas en 11 pacientes fueron preeclampsia, hipertiroidismo, diabetes gestacional e hipertensión gestacional. Discriminando las causas de hemorragia según la vía de nacimiento, el $82 \%$ fue por hipotonía uterina, hubo tres casos de retención de placenta como origen de la hemorragia obstétrica luego de parto vaginal (11,5\%), y ninguno de retención de tejido por cesárea. Hubo tres casos por trauma, uno de ellos luego de cesárea como consecuencia de un desgarro cervical luego de una prueba de instrumentación fallida. No se presentaron casos atribuibles a alteración de la coagulación.

De las 35 pacientes con HPP según la definición anotada se logró control de sangrado en 29 con manejo médico (figura 1) basado en oxitocina en dosis entre 20 y 60 UI (mediana de 40 UI). En 19 de las 29 mujeres $(65,5 \%)$ fue necesario adicionar metilergonovina a una dosis mediana de 0,2 $\mathrm{mg}$, y en 12 (41,3\%) se requirió, además de los dos medicamentos previos, el empleo de misoprostol a una 
Tabla 1.

Características demográficas de los casos de hemorragia obstétrica posparto en el Hospital de Engativá, Bogotá, Colombia, 2016

\begin{tabular}{|c|c|}
\hline Variable & $\mathrm{n}=35(100 \%)$ \\
\hline Edad* & $24(20-30)$ \\
\hline Mujeres con antecedentes médicos & $6(17)$ \\
\hline \multicolumn{2}{|l|}{ Paridad } \\
\hline Nulíparas & $19(54,3)$ \\
\hline Multíparas & $16(45,7)$ \\
\hline Edad gestacional (semanas) ${ }^{*}$ & $38(35-39)$ \\
\hline \multicolumn{2}{|l|}{ Controles prenatales } \\
\hline No & $4(11,5)$ \\
\hline Sí & $31(88,5)$ \\
\hline Concentración de hemoglobina del último control prenatal $(\mathrm{g} / \mathrm{dL})^{*}$ & $13,3(11,3-16,8)$ \\
\hline Enfermedad asociada al embarazo & $11(31,4)$ \\
\hline Altura uterina mayor a $35 \mathrm{~cm}$ & $2(11,4)$ \\
\hline Antecedente de hemorragia posparto & $0(0)$ \\
\hline Inducción del trabajo de parto & $16(45,7)$ \\
\hline \multicolumn{2}{|l|}{ Vía del nacimiento } \\
\hline Vaginal & $26(65,4)$ \\
\hline Cesárea & $9(34,6)$ \\
\hline Episiotomía $(\mathrm{n}=26)$ & $5(14,2 \%)$ \\
\hline Sangrado posparto estimado $(\mathrm{mL})(\mathrm{n}=32)^{*}$ & $800(500-1.000)$ \\
\hline \multicolumn{2}{|l|}{ Causa de hemorragia posparto } \\
\hline $\begin{array}{l}\text { Hipotonía } \\
\text { Trauma } \\
\text { Tejido } \\
\text { Coagulación }\end{array}$ & $\begin{array}{c}29(82) \\
3(8,5) \\
3(8,5) \\
0(0)\end{array}$ \\
\hline Peso de neonato $(\mathrm{g})^{*}$ & $3.024(2.630-3.490)$ \\
\hline Talla de neonato $(\mathrm{cm})^{*}$ & $51,2(49-53)$ \\
\hline
\end{tabular}

* Mediana (rango intercuartil).

dosis mediana de 1.000 microgramos por vía rectal. En las 11 pacientes en las que se activó el código rojo obstétrico la administración de medicamentos se realizó de manera simultánea.
De las restantes seis pacientes con hemorragia obstétrica que requirieron intervenciones adicionales, cuatro pacientes posparto vaginal respondieron a taponamiento hidrostático, y dos que presentaron 
Figura 1.

Flujograma de pacientes y desenlaces maternos según vía del parto y activación de código rojo obstétrico en mujeres con hemorragia obstétrica posparto en el Hospital de Engativá, Bogotá, Colombia, 2016

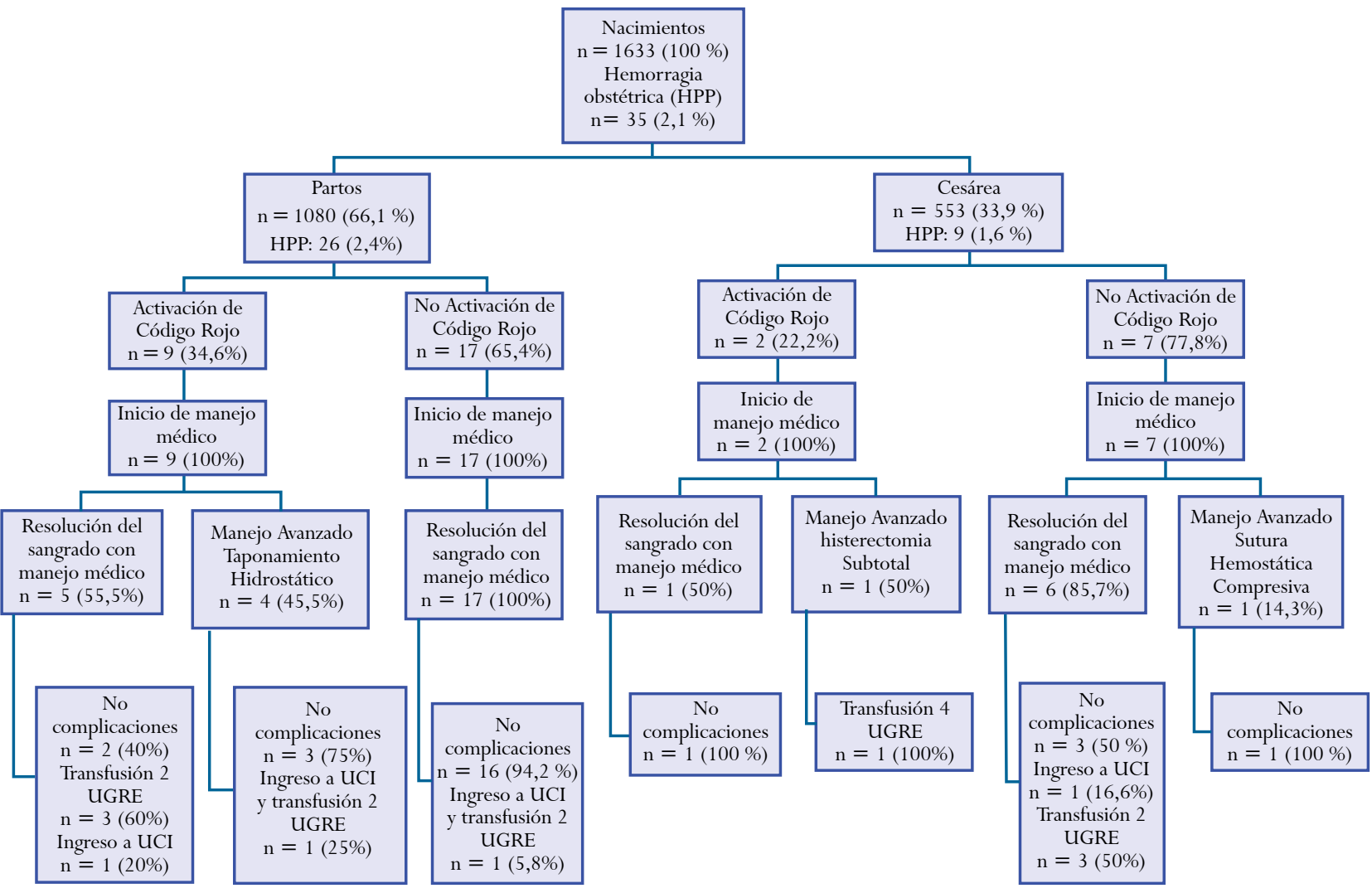

HPP: hemorragia obstétrica.

UCI: unidad de cuidado intensivo.

UGRE: unidad de glóbulos rojos empaquetados.

hemorragia obstétrica poscesárea requirieron manejo quirúrgico: una sutura hemostática (B-Lynch) y una histerectomía subtotal.

En 14 (63\%) de las 22 pacientes en las que se midió la hemoglobina luego de 6 horas del episodio de sangrado se encontró una hemoglobina menor de 11 . El $63 \%$ de mujeres en las que no se activó el protocolo de respuesta rápida presentó anemia en el puerperio; el $50 \%$ requirió transfusión de hemoderivados y el $16 \%$ requirió ingreso a UCI. El manejo médico avanzado de la hemorragia obstétrica con taponamiento hidrostático $(1,5,11)$ se utilizó en cuatro mujeres tras parto vaginal y fue efectivo en el $100 \%$.
La tabla 2 muestra la respuesta a los tratamientos instaurados según el grado de choque al momento de identificación de los casos. El análisis estratificado muestra que las mujeres con mayor grado de choque respondieron en menor proporción al manejo médico y fueron transfundidas con mayor frecuencia.

\section{DISCUSIÓN}

La incidencia de hemorragia obstétrica en el presente estudio fue de 2,1\%, menor a la reportada en la literatura mundial (4-6\%), y a la reportada previamente para la institución $(1,3,8,13)$. La incidencia establecida por el empleo de medicamentos 


\begin{tabular}{|c|c|c|c|c|}
\hline \multicolumn{5}{|c|}{$\begin{array}{l}\text { Tabla } 2 . \\
\text { Respuesta clínica según grado de choque en mujeres con hemorragia obstétrica posparto } \\
\text { en el Hospital de Engativá, Bogotá, Colombia, } 2016\end{array}$} \\
\hline $\begin{array}{l}\text { Grado de choque } \\
n=35(100 \%)\end{array}$ & $\begin{array}{l}\text { Respuesta al } \\
\text { manejo médico } \\
\text { n (\%) }\end{array}$ & $\begin{array}{l}\text { Necesidad } \\
\text { de manejo } \\
\text { avanzado* } \\
\text { n }(\%)\end{array}$ & $\begin{array}{l}\text { Necesidad } \\
\text { de manejo } \\
\text { quirúrgico } \\
\text { n }(\%)\end{array}$ & $\begin{array}{l}\text { Necesidad de } \\
\text { transfusión } \\
\quad \text { n (\%) }\end{array}$ \\
\hline $\begin{array}{l}\text { COMPENSADO } \\
\text { Frecuencia cardiaca } 60-90 \mathrm{lat} / \mathrm{min} \text {, } \\
\text { Presión arterial sistólica }>90 \mathrm{~mm} / \mathrm{Hg} \\
\mathrm{n}=17(48,5)\end{array}$ & $14(82,3)$ & $2(11,7)$ & $1 †(5,8)$ & $2(11,7)$ \\
\hline $\begin{array}{l}\text { LEVE } \\
\text { Frecuencia cardiaca } 91-100 \text { lat/min, } \\
\text { Presión arterial sistólica } \\
80-90 \mathrm{~mm} / \mathrm{Hg} \\
\mathrm{n}=5(14,2)\end{array}$ & $5(100)$ & $0(0)$ & $0(0)$ & $1(20)$ \\
\hline $\begin{array}{l}\text { MODERADO } \\
\text { Frecuencia cardiaca } 101-120 \mathrm{lat} / \mathrm{min} \text {, } \\
\text { Presión arterial } \\
\text { sistólica } 70-79 \mathrm{~mm} / \mathrm{Hg} \\
\mathrm{n}=9(25,7)\end{array}$ & $7(77,7)$ & $2(22,3)$ & $0(0)$ & $4(44,4)$ \\
\hline $\begin{array}{l}\text { SEVERO } \\
\text { Frecuencia cardiaca }>120 \mathrm{lat} / \mathrm{min}, \\
\text { Presión arterial sistólica } \\
<70 \mathrm{~mm} / \mathrm{Hg} \\
\mathrm{n}=4(11,4)\end{array}$ & $3(75)$ & $0(0)$ & $1 \div(25)$ & $2(50)$ \\
\hline
\end{tabular}

adicionales al manejo activo del alumbramiento, una vez excluidas las mujeres en las que se anotó la utilización preventiva de los uterotónicos posparto, fue 3,5 veces la incidencia reportada según la definición de caso utilizada por el Sistema de Vigilancia Epidemiológica (SIVIGILA). En las gestantes que requirieron el uso adicional de uterotónicos se presentó morbilidad materna y requirieron transfusión en un $50 \%$ de los casos y manejo invasivo en el 16\%. Esta observación sugiere que podría ser conveniente hacer el diagnóstico de HPP mediante la utilización de métodos más repetibles, como uso de medicamentos uterotónicos, y evaluar diferentes puntos de corte de acuerdo con la necesidad progresiva de estos.
La cesárea se ha reportado como un factor de riesgo, y se describe una incidencia mayor de HPP en mujeres con esta vía del parto (12). Sin embargo, en el presente estudio la incidencia de HPP poscesárea fue menor comparada con la incidencia tras parto vaginal (1,6\% vs. 2,4\%). Estas diferencias pueden deberse a la exclusión de los casos con causas de HPP identificados anteparto como placenta previa, acretismo placentario, etc., y por la población incluida con características de bajo riesgo para HPP.

La baja frecuencia de activación del código rojo refleja las dificultades para el diagnóstico clínico de la hemorragia obstétrica severa por el personal responsable de la atención del parto $(13,14)$. La estimación visual del sangrado fue evaluada en una 
revisión de la literatura donde se incluyeron 36 estudios (14), y se observó que la apreciación clínica no era precisa al compararla con bolsas de recolección. Sin embargo, el empleo de estas últimas no previno la demora en la toma de decisiones ni la progresión de la hemorragia obstétrica. Los falsos positivos de este método de estimación del sangrado estimado tendrían como ventaja llevar a manejos tempranos en pacientes con sangrados no severos pero anormales (13) y, a juicio del personal tratante, podría evitar la activación del protocolo institucional, la notificación y el uso excesivo de recursos ante esta emergencia obstétrica. Por el contrario, un falso negativo lleva a inicio tardío del manejo por una subestimación del sangrado que puede conllevar consecuencias graves que incrementan la morbilidad para la gestante.

Nuestras observaciones respecto a las causa de HPP concuerdan con un estudio realizado en países de bajos ingresos, que reportó que la atonía uterina fue la causante del $82 \%$ de las hemorragias obstétricas (15).

El tratamiento protocolizado de la hemorragia obstétrica se ha consolidado como una secuencia de intervenciones que van ascendiendo en complejidad y grado de invasión $(5,11,12)$. El éxito del manejo médico inicial con medicamentos uterotónicos y masaje se reporta entre un 60-70\% de los casos (6). En el presente estudio, el manejo médico tras la activación del código rojo obstétrico fue exitoso en 6/11 (54,5\%), mientras que fue de 29/35 (82\%) incluyendo a las mujeres en las que no se activó el código rojo obstétrico. Esta diferencia podría deberse, como se ha mencionado, a diferencias en la magnitud o la severidad del sangrado que motivan al personal tratante a la activación selectiva del protocolo institucional: en las mujeres con sangrado estimado mayor se llevan a cabo las conductas coordinadas preestablecidas y opciones de manejo avanzado o quirúrgico de manera expedita pero con inicio tardío, mientras que en las mujeres con sangrado anormal que motivó el uso de uterotónicos adicionales al manejo activo del alumbramiento, sin activación del código rojo, la respuesta a dicho tratamiento médico es cercana al 80 \%. En este mismo sentido, el análisis estratificado de la respuesta de las mujeres al tratamiento instaurado en relación con el grado de choque mostró que aquellas con mayor grado de choque respondieron en menor proporción al manejo médico y fueron transfundidas con mayor frecuencia.

La necesidad de transfusión e ingreso a UCI en pacientes con hemorragia en las que no se activó el código rojo obstétrico sugieren la necesidad de evaluar el impacto de la identificación y activación del protocolo de respuesta rápida a la hemorragia obstétrica disparado por el uso de algún medicamento uterotónico específico, o por el empleo de más de uno de estos medicamentos, en la medida que aún en hemorragias consideradas no severas, dada la pobre validez de la estimación clínica del sangrado y la tardanza hasta el hallazgo de signos de choque (13), existe repercusión sobre la respuesta al tratamiento y sobre la salud materna.

La efectividad del taponamiento hidrostático encontrada en nuestra serie de casos concuerda con la reportada en diversos estudios observacionales donde se informa que el taponamiento hidrostático resuelve entre el 74 al $90 \%$ de las hemorragias obstétricas que no responden a manejo médico durante el parto vaginal (17-20), y está recomendado como primera línea de manejo avanzado cuando la causa del sangrado es la atonía (12). En las mujeres que tuvieron hemorragia obstétrica poscesárea, el tratamiento de segunda línea realizado fue inmediatamente quirúrgico, en concordancia con las recomendaciones de la OMS (5), y por la disponibilidad del recurso quirúrgico en la institución. Este hallazgo también está relacionado con el protocolo institucional vigente (11) que recomienda que las mujeres con grados severos de choque, según los parámetros establecidos en la guía, sean sometidas a tratamiento quirúrgico con prontitud y se reanimen de manera pronta con hemoderivados.

El presente trabajo tiene como fortalezas la identificación activa de los casos mediante fuentes 
confiables como el despacho de medicamentos desde la farmacia y que permiten el control de sesgos de medición, reporte y mala clasificación. Lo anterior permitió identificar las pacientes con hemorragia obstétrica que no habrían sido detectadas por activación del equipo de respuesta rápida o por la necesidad de transfusión de hemoderivados. El estudio tiene limitaciones por ser de carácter observacional, basado en registros, y por la baja incidencia observada del evento.

\section{CONCLUSIONES}

Los hallazgos del presente estudio revelan la necesidad de evaluar la efectividad y seguridad de métodos diagnósticos más reproducibles para definir el momento oportuno para iniciar el tratamiento protocolizado en las mujeres que presentan hemorragia posparto severa, independientemente de la vía del parto, e incentivar la activación del equipo de respuesta rápida de la hemorragia, ante la evidencia de morbilidad y desenlaces maternos adversos en mujeres en los que no se activó el código rojo obstétrico.

\section{REFERENCIAS}

1. American College of Obstetricians and Gynecologists. ACOG Practice Bulletin: Clinical Management Guidelines for Obstetrician-Gynecologists Number 76, October 2006: postpartum hemorrhage. Obstet Gynecol. 2006;108:1039-47.

2. Say L, Chou D, Gemmill A, Tunçalp Ö, Moller A-B, Daniels J, et al. Global causes of maternal death: A WHO systematic analysis. Lancet Glob Health. 2014;2:e323-333. https://doi.org/10.1016/S2214$109 \mathrm{X}(14) 70227-\mathrm{X}$

3. Carroli G, Cuesta C, Abalos E, Gulmezoglu AM. Epidemiology of postpartum haemorrhage: A systematic review. Best Pract Res Clin Obstet Gynaecol. 2008;22:999-1012. https://doi.org/10.1016/j.bpobgyn.2008.08.004

4. Francois KE, Foley MR. Antepartum and pospartum hemorrhage. En: Gabbe SG, Niebyl JR, Simpson JL, Landon MB, Galan HL, Jauniaux ERM, et al, Editors. Obstetrics. Normal and Problem Pregnancies. Seventh edition; 2017. p. 395-424.
5. WHO Recommendations for the Prevention and Treatment of Postpartum Haemorrhage. Geneva: World Health Organization; 2012. (WHO Guidelines Approved by the Guidelines Review Committee) [visitado 2017 Sep 15]. Disponible en: http://www.ncbi. nlm.nih.gov/books/NBK131942/

6. OB Hemorrhage Toolkit V 2.0 | California Maternal Quality Care Collaborative [visitado 2017 Sep 15]. Disponible en: https://www.cmqcc.org/resourcestool-kits/toolkits/ob-hemorrhage-toolkit

7. Tsu VD. Postpartum haemorrhage in Zimbabwe: a risk factor analysis. Br J Obstet Gynaecol. 1993;100:327-33. https://doi.org/10.1111/j.1471-0528.1993.tb12974.x

8. Al-Zirqi I, Vangen S, Forsen L, Stray-Pedersen B. Prevalence and risk factors of severe obstetric haemorrhage. BJOG Int J Obstet Gynaecol. 2008;115:1265-72. https://doi.org/10.1111/j.1471-0528.2008.01859.x

9. Ayadi AME, Nathan HL, Seed PT, Butrick EA, Hezelgrave NL, Shennan AH, et al. Vital sign prediction of adverse maternal outcomes in women with hypovolemic shock: The role of Shock Index. PLOS ONE. 2016;11:e0148729. https://doi.org/10.1371/journal. pone.0148729

10. PRO Morbilidad materna extrema.pdf [visitado 2017 Sep 15]. Disponible en: Vigilancia/sivigila/ Protocolos \%20SIVIGILA/PRO \%20Morbilidad \%20 Materna \%20Extrema.pdf

11. Vélez-Álvarez GA, Agudelo-Jaramillo B, Gómez-Dávila JG, Zuleta-Tobón JJ. Código rojo: guía para el manejo de la hemorragia obstétrica. Rev Colomb Obstet Ginecol. 2016;60:34-48.

12. Postpartum haemorrhage, prevention and management (Green-top Guideline No. 52). Royal College of Obstetricians and Gynaecologists [visitado 2017 Sep 15]. Disponible en: https://www.rcog.org.uk/en/ guidelines-research-services/guidelines/gtg52/

13. Rubio-Romero JA, Guevara-Cruz ÓA, Gaitán-Duarte H. Validity of visual estimation of blood loss as diagnostic tool in severe post partum hemorrhage in an university hospital. Bogotá. 2007. Rev Fac Med. 2010;58:173-84.

14. Rubio-Romero JA, Gaitán-Duarte HG, RodríguezMalagón N. Concordancia entre la estimación visual y la medición del volumen recolectado en una bolsa del sangrado intraparto en mujeres con parto normal en Bogotá, Colombia, 2006. Rev Colomb Obstet Ginecol. 2016;59:92-102. 
15. Ngwenya S. Postpartum hemorrhage: Incidence, risk factors, and outcomes in a low-resource setting. Int J Womens Health. 2016;8:647-50. https://doi. org/10.2147/IJWH.S1 19232

16. Jiménez-Hernández DL, Guevara-Rodríguez AP, Zuleta-Tobón JJ, Rubio-Romero JA. Tasa de cesáreas por grupos de Robson en una institución de mediana complejidad de la ciudad de Bogotá, 2012-2014. Rev Colomb Obstet Ginecol. 2016;67:101-11. https://doi. org/10.18597/rcog.381

17. Kandeel M, Sanad Z, Ellakwa H, El Halaby A, Rezk M, Saif I. Management of postpartum hemorrhage with intrauterine balloon tamponade using a condom catheter in an Egyptian setting. Int J Gynaecol Obstet off Organ Int Fed Gynaecol Obstet. 2016;135:272-5. https://doi.org/10.1016/j.ijgo.2016.06.018
18. Goldrath MH. Uterine tamponade for the control of acute uterine bleeding. Am J Obstet Gynecol. 1983;147:869-72. https://doi.org/10.1016/00029378(83)90237-5

19. Keriakos R, Mukhopadhyay A. The use of the Rusch balloon for management of severe postpartum haemorrhage. J Obstet Gynaecol J Inst Obstet Gynaecol (i). 2006;26:335-8. https://doi. org/10.1080/01443610600595077

20. Condous GS, Arulkumaran S, Symonds I, Chapman R, Sinha A, Razvi K. The "tamponade test" in the management of massive postpartum hemorrhage. Obstet Gynecol. 2003;101:767-72. 